\title{
NICS-TNG infrared spectroscopy of trans-neptunian objects 2000 EB173 and 2000 WR106
}

\author{
J. Licandro ${ }^{1}$, E. Oliva ${ }^{1,2}$, and M. Di Martino ${ }^{3}$ \\ 1 Centro Galileo Galilei \& Telescopio Nazionale Galileo, PO Box 565, 38700, S/C de La Palma, Tenerife, Spain \\ 2 Osservatorio di Arcetri, Largo E. Fermi 5, 50125 Firenze, Italy \\ 3 Osservatorio Astronomico di Torino, 10025 Pino Torinese, Italy
}

Received 12 April 2001 / Accepted 25 May 2001

\begin{abstract}
We report complete near-infrared $(0.9-2.4 \mu \mathrm{m})$ spectral observations of trans-neptunian objects (TNOs) 2000 EB173 and 2000 WR106 collected using the new Near Infrared Camera Spectrometer (NICS) attached to the $3.56 \mathrm{~m}$ Telescopio Nazionale Galileo (TNG). Both spectra are very red and with a quite strong and broad drop extending throughout the $K$ band. However, while 2000 EB173 does not show any evidence of narrow absorption features, the spectrum of 2000 WR106 has quite deep water ice absorption at 1.5 and $2.0 \mu \mathrm{m}$. Moreover, the latter object is significantly less red than the former indicating, therefore, that the surface of 2000 WR106 is "cleaner" (i.e. less processed by particle irradiation) than that of 2000 EB173.
\end{abstract}

Key words. minor planets - comets - infrared - trans-neptunian objects

\section{Introduction}

The population of objects in the region just beyond the orbit of Neptune (TNOs), called the Edgeworth-Kuiper belt (EKb), are remnant planetesimals from the early solar system formation stages (Edgeworth 1949; Kuiper 1951). They probably contain some of the least modified materials remaining from the protosolar nebula, though the study of their surface properties is very important from a cosmogonical point of view. Their study could provide important clues to understand the conditions existing at the beginning of the solar system.

Near-infrared spectroscopy is a powerful mean for remote determination of the composition of volatile surface component of the outer solar system objects (Brown \& Cruikshank 1997), but, due to the small size and distance of this objects, this technique is actually limited to the brighter members of the EKb. In fact, only 4 TNOs have published near-infrared spectra, and all obtained with the Keck $10 \mathrm{~m}$ telescope. Brown et al. (1997) present the spectra of 1993 SC; Luu \& Jewitt (1998) the spectra of 1996 TL66; Brown et al. (1999) the one of 1996 TO66; and finally Brown et al. (2000) the spectra of 2000 EB173. Among them, only for 1996 TL66 the spectrum spans the whole near-infrared range, while for the others the spectra cover the $H$ and $K$ bands $(1.4-2.4 \mu \mathrm{m})$ only. Even though there are very few TNO spectra published,

Send offprint requests to: J. Licandro, e-mail: licandro@tng.iac.es different surface properties have been inferred among them. The spectra of $1993 \mathrm{SC}$ show absorption bands possibly due to hydrocarbons, the one of 1996 TO66 shows absorptions due to water ice, while the spectra of 1996 TL66 and 2000 EB173 are featureless in the observed range. This diversity of surface compositions has been observed also in similar objects like Centaurs and irregular satellites (Brown 2000).

During the commissioning phase of NICS at TNG, we had the opportunity to observe two of the brighter known TNOs: 2000 EB173 and 2000 WR106 $(V=19.7$ and 20.1, respectively). Assuming a geometric albedo of $4 \%$, these objects have diameters of 670 and $1100 \mathrm{~km}$ respectively. If the typically assumed albedo is correct, 2000 WR106 is the larger known TNO after Pluto. From the dynamical point of view, the 2000 EB173 orbital elements suggest that the orbit is compatible with a Pluto-like 2:3 resonance, and as Pluto, it is a Neptune-crosser. Its orbital elements are very similar to those of TNO 1995 QY9. On the other hand, 2000 WR106 has orbital elements that fit with those of the classical TNO group.

\section{Observations}

We have obtained low resolution spectra of 2000 WR106 and 2000 EB173 on January 14 and February 5, 2001 respectively, with the $3.6 \mathrm{~m}$ Telescopio Nazionale Galileo (TNG) during the commissioning phase of NICS, the near-infrared camera and spectrometer expressly designed and built for the TNG. NICS is a FOSC-type cryogenic 
focal reducer, equipped with two interchangeable cameras feeding a Rockwell Hawaii $1024 \times 1024$ array. The camera used for the spectroscopic observations has a focal ratio of $\mathrm{F} / 4.3$, corresponding to a plate scale $=0.25^{\prime \prime} /$ pixel (Oliva \& Gennari 1995; Baffa et al. 2000). Among the many imaging and spectroscopic observing modes, NICS offers a unique, high throughput, low resolution spectroscopic mode with an Amici prism disperser (Oliva 2001), which yields a complete $0.9-2.5 \mu \mathrm{m}$ spectrum. A $1.0^{\prime \prime}$ width slit corresponding to a spectral resolving power $R \simeq 50$ and quasi-constant along the spectrum, has been used. The low resolution together with the high efficiency of the Amici prism (about 90\% in all the infrared range) allowed us to obtain spectra of faint objects like TNOs with a four meter class telescope for the first time, and with the advantage of having the whole infrared range measured simultaneously.

The identification of the TNOs was done by taking images through the $J_{\mathrm{s}}$ filter the day of the observations and the day before, and by comparing them. The objects were identified as moving objects at the predicted position and with the predicted proper motion. The slit was oriented in the direction of the motion of the TNOs, so they remained in the slit during the integration tracking at the sidereal rate. The acquisition consisted of a series of 3 images of $90 \mathrm{~s}$ exposure time in one position (position $A)$ of the slit for $2000 \mathrm{~EB} 173(4 \times 60 \mathrm{~s}$ for $2000 \mathrm{WR} 106)$ and then offsetting the telescope by $40^{\prime \prime}$ in the direction of the slit (position $B$ ). This process was repeated and a number of $A B B A$ cycles were acquired. The total exposure time was $3360 \mathrm{~s}$ for $2000 \mathrm{WR} 106$, and $4320 \mathrm{~s}$ for 2000 EB173 respectively. The reduction of the spectra was done by subtracting consecutive $A$ and $B$ images. Each $A-B$ frame had some residual of sky emission, related to sky transparency fluctuations and/or intrinsic variation of the air-glow atmospheric emission, which was eliminated by extracting the "short-slit" spectrum around each $A$, $B$ spectrum, then aligning and combining them into the final short-slit spectrum from which the 1D spectra were extracted. No contribution from residual sky emission was found within the noise of the spectra. Wavelength calibration was performed using an argon lamp and the deep telluric absorption features.

To correct for telluric absorption, A0 stars were observed during the same night (SAO 058110 and SAO 042804 on January 14, and SAO 058110, SAO 042804, and SAO 018946 on February 5). The spectra of the TNOs were divided by the spectra of the A0 stars, multiplied by the ratio of the blackbody function of the A0 stars, divided by that of the Sun, and then normalized to unity at $1.7 \mu \mathrm{m}$, thus obtaining the relative reflectance plotted in Fig. 1. Around the telluric water band absorptions the $S / N$ of the spectrum is very strong, and also this absorption varies between the TNO spectra and the standard stars spectra introducing false spectral features. Therefore, these parts are not included in the final spectrum. Finally, the $J$ magnitude of 2000 EB173 was obtained by means of aperture photometry on the $J_{\mathrm{s}}$ images $(J=18.2 \pm 0.11)$.

\section{Discussion}

The spectra of both objects are shown in Fig. 1 together with the reflectance spectrum of $10 \mu \mathrm{m}$ water ice grains at $90 \mathrm{~K}$, taken from Brown \& Koresko (1998). Both TNOs spectra present a very red slope between 0.9 and $1.8 \mu \mathrm{m}$, equivalent to $J-H=0.7$ and $J-H=0.8$ for 2000 WR106 and 2000 EB173, respectively. But the slope drastically changes in the $K$ band. The turn over between 1.6-1.8 $\mu \mathrm{m}$ and subsequent depression extending throughout the $K$ band is more conveniently visualized in the smoothed spectra displayed in Fig. 2, where one can also notice that the two objects have remarkably different spectral behavior within the $H$ and $K$ bands.

The spectrum of 2000 WR106 shows absorption features at 1.5 and $2.0 \mu \mathrm{m}$ typically produced by water ice in the surface. On the other hand, the absorption band observed in the $K$ region in the spectrum of 2000 EB173 is much more extended and flat. Also no signs of any absorption at $1.5 \mu \mathrm{m}$ is detected. We should conclude that water ice has not been observed in 2000 EB173 surface. This is in agreement with Brown et al. (2000), who present a spectrum of $2000 \mathrm{~EB} 173$ in the $1.4-2.4 \mu \mathrm{m}$ region that is similar to our in the same bands. They also conclude that the spectrum is featureless, but the additional information provided by our spectrum in the $0.9-1.3 \mu \mathrm{m}$ region shows that, in the near-IR spectral range, absorption features exist.

The observed very red color of both objects is probably due to an evolved surface which is the result of long term irradiation by solar radiation, solar wind, and galactic cosmic-rays. This results in the selective loss of hydrogen and the formation of an "irradiation mantle" of carbon residues (Moore et al. 1983; Johnson et al. 1984; Strazzulla \& Johnson 1991). This process makes that an initially neutral color and high albedo ice becomes redish. Further irradiation gradually reduces the albedo at all wavelengths, and the material becomes very dark, neutral in color, and spectrally featureless (Andronico et al. 1987; Thompson et al. 1987). The surface water ice present in 2000 WR106 and the fact that it is less red than the surface of 2000 EB173 suggest that the thickness of 2000 WR106 irradiation mantle is smaller than that of 2000 EB173. There are two possible explanations for this: the irradiation processes in 2000 EB173 were more intense than in 2000 WR106 thus more "fresh" original material remains in the surface; or the surface of 2000 WR106, was more affected by collisions. In the second case, collisions affected deeper layers of the irradiation mantle of 2000 WR106 than of 2000 EB173, reaching "fresh" ice under the irradiation mantle (Gil-Hutton, personal communication). This fresh material can be also affected by collisions (Hartmann 1980).

It is also very interesting to note that the turn-over in $H$ and drop in $K$ seems to be usual in the spectra of similar red objects. That is the case of Centaurs 8405 Asbolus (Barucci et al. 2000; Kern et al. 2000), 5145 Pholus (Cruikshank et al. 1998), and TNOs $1996 \mathrm{TP}_{66}$ 


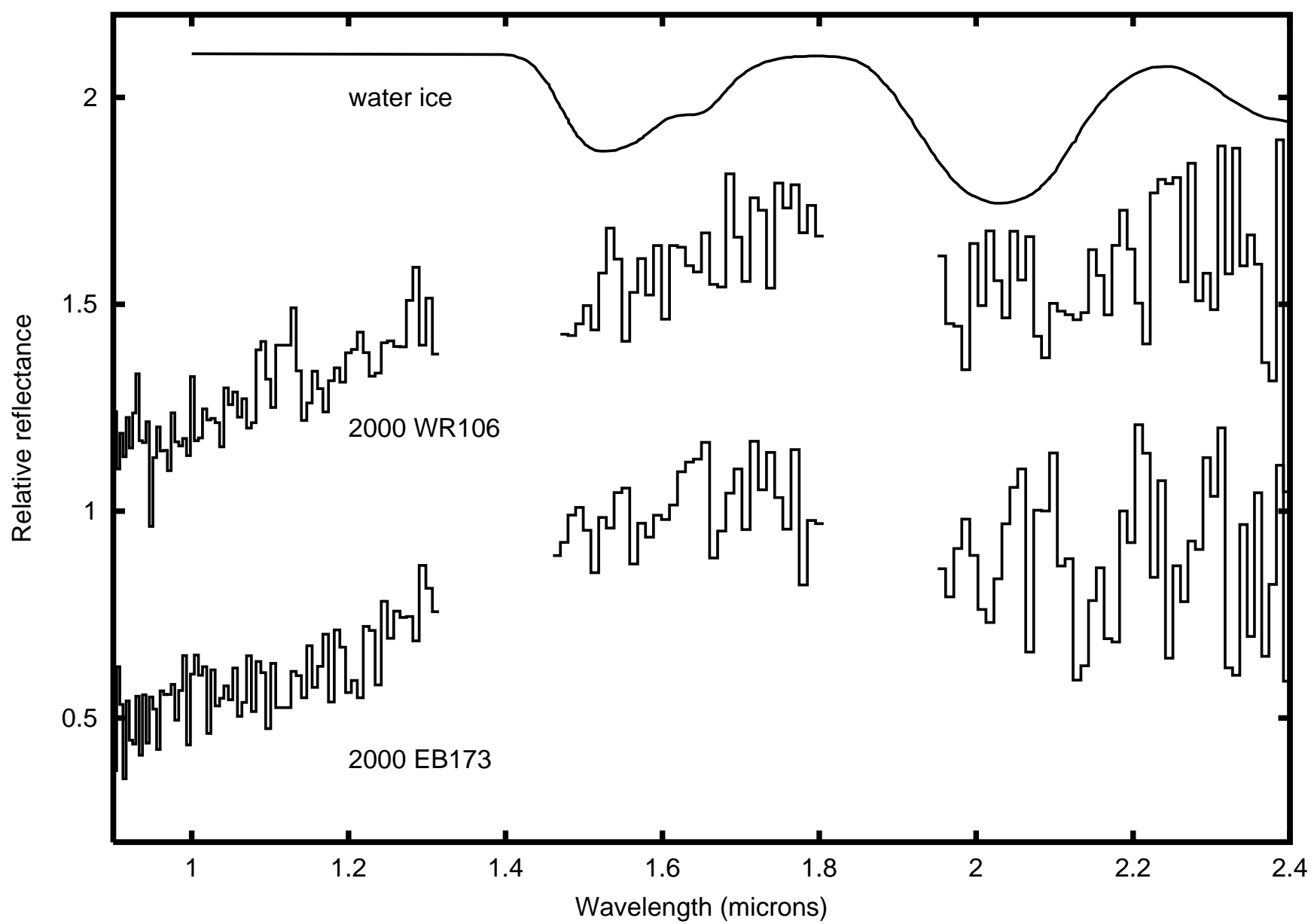

Fig. 1. Reflectance spectra of 2000 EB173 and 2000 WR106. The spectra has been normalized at $1.7 \mu \mathrm{m}$. The spectrum of 2000 WR106 is shifted. The reflectance spectrum of $10 \mu \mathrm{m}$ water ice grains at $90 \mathrm{~K}$, taken from Brown \& Koresko (1998) is also included. Note the 1.5 and $2.0 \mu \mathrm{m}$ water ice absorption bands on the spectrum of $2000 \mathrm{WR} 106$.

and $1996 \mathrm{TS}_{66}$ (Noll et al. 2000). In some cases, like 5145 Pholus and 2000 WR106, water ice absorption bands could be in principle considered as the most important contributor to the observed turn over. But is it not the case of 2000 EB173. Also Centaur 8405 Asbolus is a very interesting case, as it presents a discrete region composed mostly of water ice superposed on a darker surface according to Kern et al. (2000). They observed that the spectrum of this object $(1.1-1.9 \mu \mathrm{m})$ varies during the 1.7 hours of the observations. At the beginning a broad absorption band at $1.6 \mu \mathrm{m}$ is clearly detected (corresponding to the spot of water ice) and dissapears in the late three spectra, when the water ice spot is not visible. But the change in the slope at about $1.7 \mu \mathrm{m}$ remains. Barucci et al. (2000) also observed this turn over, but not the typical water ice absorption bands, and show that 8405 Asbolus also presents a red slope in the $0.4-1.7 \mu \mathrm{m}$ region. So, in this very red object, even in the absence of water ice, the change of the slope of the spectrum in the $H$ band is very pronounced. We should conclude that there is a strong and very extended absorption band in the $K$ region in all observed objects that present an irradiation mantle. It should be expected that the materials that produce this absorption probably originate from the same process. Materials like Titan tholin and nitrogen-bearing molecules can contribute significatively (see Cruikshank et al. 1998). More modeling of the observed spectra and laboratory experiments are needed.

Finally, low resolution spectroscopy in the whole 0.9-2.4 $\mu \mathrm{m}$ range of a significant number of TNOs and Centaurs is crucial for the understanding of their surface properties and the physical process that control their evolution.

Acknowledgements. We thank Dale P. Cruikshank for his useful comments on the manuscript and Leonardo Testi for his help in the reduction of the spectra.

This paper is based on observations made with the Italian Telescopio Nazionale Galileo (TNG) operated on the island of La Palma by the Centro Galileo Galilei of the CNAA (Consorzio Nazionale per l'Astronomia e l'Astrofisica) at the Spanish Observatorio del Roque de los Muchachos of the Instituto de Astrofisica de Canarias. We are grateful to all the technical staff and telescope operators for their assistance during the commissioning phase of NICS. 


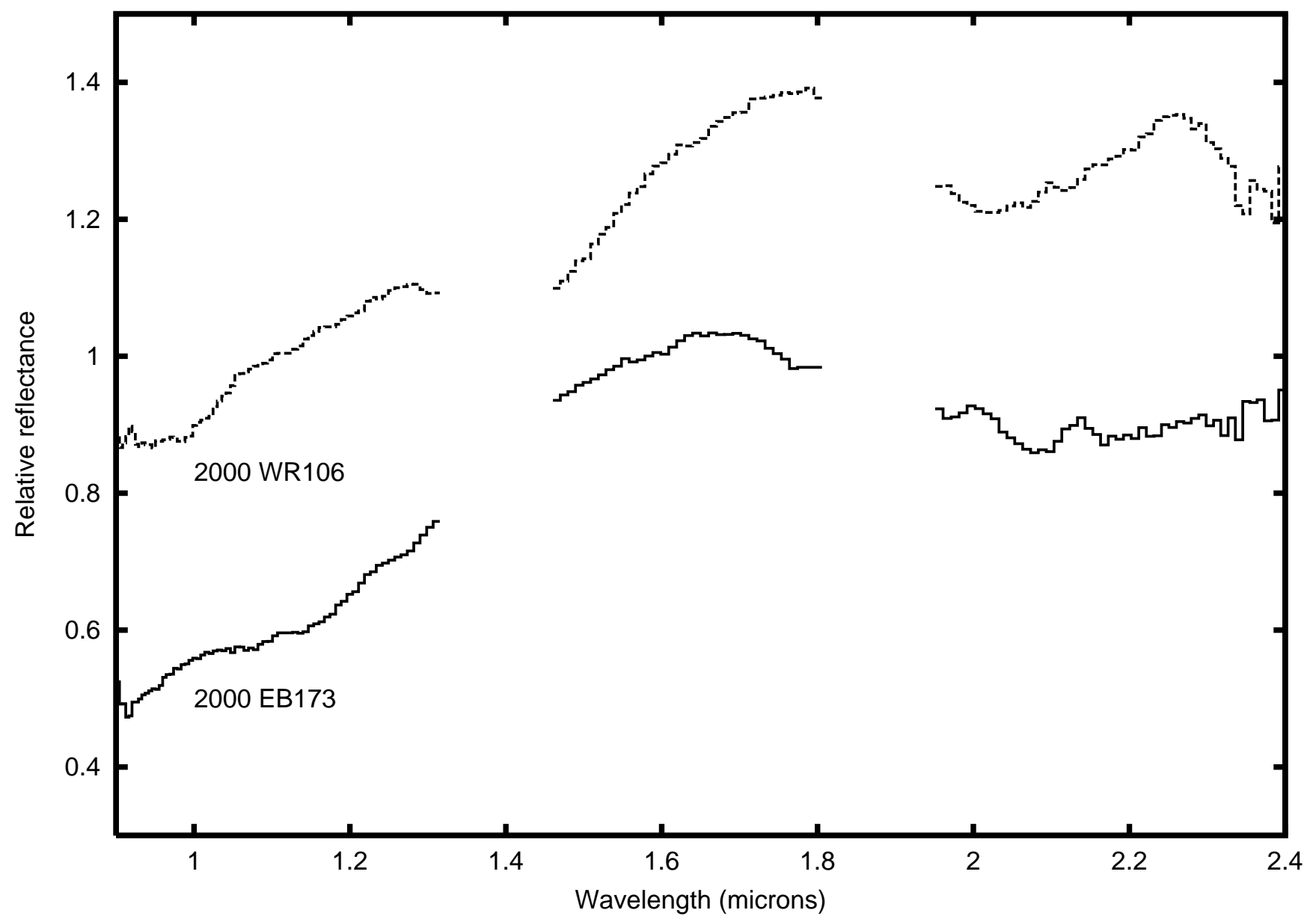

Fig. 2. Smoothed version (box-car smoothing of 15 pixels) of the spectra shown in Fig. 1. Note that both spectra present strong absorptions in the $K$ band. 2000 WR106 spectrum also shows signatures of water ice in the surface, at 2.0 and $1.5 \mu \mathrm{m}$.

\section{References}

Andronico, G., Baratta, G., Spinella, F., \& Strazzulla, G. 1987, A\&A, 184, 333

Barucci, M., Bergh, C., Cuby, J., et al. 2000, A\&A, 357, L53 Baffa, C., Gennari, S., Lisi, F., et al. 2000, in The Scientific dedication of the Telescopio Nazionale Galileo, Conference held in Santa Cruz del La Palma on November 3-5, 2000 Brown, M. 2000, AJ, 119, 977

Brown, R., \& Cruikshank, D. 1997, Ann. Rev. Earth Planet. Sci., 25, 243

Brown, M., \& Koresko, C. 1998, ApJ, 505, L65

Brown, R., Cruikshank, D., Pendleton, Y., \& Veeder, G. 1997, Science, 276, 937

Brown, R., Cruikshank, D., \& Pendleton, Y. 1999, ApJ, 519, L101

Brown, M., Blake, G., \& Kessler, J. 2000, AJ, 543, L163

Cruikshank, D., et al. 1998, Icarus, 135, 389
Edgeworth, K. 1949, MNRAS, 109, 600

Hartmann, W. 1980, Icarus, 44, 441

Johnson, R., Lanzerotti, L., \& Brown, W. 1984, Adv. Space Res., 4, 41

Kern, S., McCarthy, D., Buie, M., et al. 2000, ApJ, 542, L155 Kuiper, G. 1951, in Astrophysics: A Topical Symposium, ed. J. A. Hynek (New York, McGraw Hill), 357

Luu, J., \& Jewitt, D. 1998, ApJ, 494, L117

Moore, M., Donn, B., Khanna, R., \& A'Hearn, M. 1983, Icarus, 54,388

Noll, K., Luu, J., \& Gilmore, D. 2000, AJ, 119, 970

Oliva, E. 2001, Mem. SAIt., in press [astro-ph/99109108]

Oliva, E., \& Gennari, S. 1995, A\&AS, 114, 179

Strazzulla, G., \& Johnson, R. 1991, in Comets in the PostHalley era, ed. R. L. Newburn Jr., M. Neugebauer, \& J. Rahe (Kluwer Academic Publishers, Netherlands), 243

Thompson, W., Murray, B., Khare, B., \& Sagan, C. 1987, J. Geophys. Res., 92, 14933 